\title{
Long-Term Evaluation of a Telepresence Robot for the Elderly: Methodology and Ecological Case Study
}

\author{
Amedeo Cesta $^{1}$ - Gabriella Cortellessa ${ }^{1}$ - Andrea Orlandini ${ }^{1}$ - Lorenza Tiberio $^{1}$
}

Accepted: 6 January 2016 / Published online: 20 January 2016

(C) The Author(s) 2016. This article is published with open access at Springerlink.com

\begin{abstract}
Telepresence robotic systems are proposed in different contexts and specifically in the area of social robotics for assisting older adults at home. Similarly to other robotic systems, such robots are often designed and then evaluated in laboratory settings for a limited period of time. Lab-based evaluations present limitations because they do not take into account the different challenges imposed by the fielding of robotic solutions into real contexts for longer periods. In order to perform long-term experiments in real ecological settings it is very important to define a structured approach to assess the impact of a prolonged and constant use of the telepresence robot. This paper proposes a methodology in the area of elderly people support, called MARTA, for Multidimensional Assessment of telepresence $\boldsymbol{R}$ obo $\boldsymbol{T}$ for older Adults. It introduces the main variables of interest as well as the instruments and administration timeline for assessing relevant changes that may occur over time. MARTA is also validated in a one year-long case study
\end{abstract}

Lorenza Tiberio was a Ph.D. student at Consiglio Nazionale delle Ricerche-Istituto di Scienze e Tecnologie della Cognizione (CNR-ISTC) when this research was conducted.

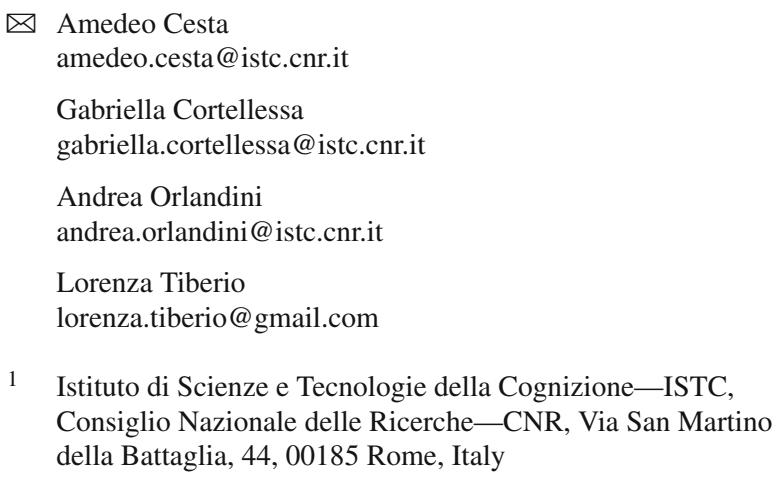

during which a telepresence robot, called GIRAFF, has been deployed and iteratively assessed. The paper also provides remarks on the technology readiness and suggestions for its improvements.

Keywords Telepresence robots - Long-term evaluation methodologies · Deployed systems $\cdot$ User needs

\section{Introduction}

The area of social robotics as well as the task of "robot as companions" has received major attention at research level since early work like [1]. Several projects have also proposed different types of solutions with robots that both interact with humans and are connected to heterogeneous technology to build innovative living environments (e.g., [2-7]).

In this work we describe part of our experience in fielding a telepresence robot in a real context of usage for a long period of time. Telepresence systems are currently available on the market, but their use within realistic ecological environments (i.e., real houses) for prolonged time of interaction is still an under-addressed issue. As a first necessary task the paper defines a methodology for evaluating the long time span of the user experience.

This work promotes the idea of continuously using a telepresence robot to support and foster the social participation of elderly people. Specifically, the study is focused on the analysis of attitude and acceptance of people who share the environments in which the telepresence robot operates over long periods of time with the aim to achieve a deeper understanding of the environmental, technical and psychological factors that can influence the adoption and effective use of a telepresence robot in a real context of daily life. This has been realized by inserting a telepresence robot into a real 
house of older users for a period of time of one year with the aim to investigate its adaptivity and compatibility in helping and supporting their social participation and interaction.

A key requirement for social companions (e.g., robots assisting old people at home) is their ability to ensure a continuous operation, their robustness and the continuous interaction with humans and environment over time. Such continuity of use has significant implications on the technology development but it also highlights the need to assess human reactions with respect to the prolonged use of the proposed solutions. It is worth underscoring that the challenges for the Intelligent Technology and the Human Robot Interaction researchers are mainly related to two aspects: (a) in terms of users perspective, robots must adhere to user needs and be acceptable in the long-term, (b) in terms of technology, the need exists to create robust, efficient and secure solutions. The proposed evaluation methodology for the system assessment takes into account environmental, technical and psychological factors to study users attitude and acceptance, a protocol for the deployment of telepresence robots technology in real contexts of use and finally a long-term ecological case study involving a couple of older users and their son for an experience of use of one year. The paper results demonstrate how long-term experience of use and ecological evaluation are key points to ensure that robotic technology can make a leap forward and be used in real environments.

Plan of the Paper The paper is organized as follows: Section 2 introduces the context of the work; Section 3 provides a discussion on related works on long-term assessment of robotic solutions; Section 4 describes the proposed methodological approach to the long-term evaluation of telepresence robots and constitutes the main contribution of this work; Section 5 illustrates an implementation of the proposed methodology on a one-year case study performed in a real house; Section 6 discusses some practical implications emerged from the case study; a concluding section provides final remarks.

\section{Context of Work}

Telepresence robots have been proposed to be used in workplace and have been studied as a means to enable remote collaboration among co-workers [8,9]. Telepresence systems are also being used to provide support to elderly people. In this respect, some research exists which aims to understand the acceptance of older adults, their concerns and attitude toward the adoption of telepresence robotic solutions [8-10].

Our work stems from this interest in understanding the users attitude and acceptance of robotic technology, a topic already addressed in one of our previous project named ROBOCARE $[3,11]$ which aimed at building an integrated sys- tem composed of sensors, intelligent software and a robotic prototype platform for supporting the older persons. One of the lessons learned from the ROBOCARE project was that the technology should be robust, simple and effective in order to be used in a real settings and in view of devising fielded study in real and ecological context of use.

The interest in testing the technology into the field, has been reinforced during our participation in an AAL (Ambient Assisted Living) project, called EXCITE ${ }^{1}$ (Enabling Social Interaction through Embodiment), aiming at promoting the use of telepresence robots to foster interaction and social participation of older people as well as to provide an easy means for possible caregivers to visit and interact with their assisted persons. The main idea of ExCITE, has been to deploy a telepresence robot (GIRAFF) in real houses and iteratively assess it in three European countries. A general description of the project goals is given in [12] while [13] also introduces an initial selection of the variables for conducting longitudinal evaluation of the robot. Within the EXCITE project the authors have been responsible for the evaluation activities of the commercial mobile telepresence platform. A key step of their activity consisted in conceiving an evaluation plan for accessing the robotic platform that takes into account the long-term impact of the technology on the user experience. This key step is described in this paper together with a complete validation of the procedure consisting of a one year trial in a real house where two older adults lived.

The GIRAFF robot and its functionalities. GIRAFF is a robot produced by GIRAFF Technologies $\mathrm{AB}^{2}$, Sweden, (see Fig. 1a). It is a remotely controlled mobile, human-height physical avatar integrated with a videoconferencing system (including a camera, display, speaker and microphone). It is powered by motors that can propel and turn the device in any direction. An LCD panel is incorporated into the head unit (Fig. 1b). The robotic platform is accessed and controlled via a standard computer/laptop using a software application. From a remote location a member of family or healthcare professionals (secondary user), even with limited prior computer training, teleoperates the robotic platform while older people (primary users) living in their own home (where the robot is placed) can receive their visit through the telepresence robot. The primary user can accept or reject a call via the buttons on the robot (Fig. 1c) or alternatively using the remote control (Fig. 1e). The remote user can charge the robot by driving it onto a docking station (Fig. 1d). The robot is not endowed with any autonomous capabilities, i.e., the robot's control is fully tele-operated by a secondary user and does not show any proactive behavior.

Both the primary and secondary users can take advantage from the deployment of the robot in home environments.

\footnotetext{
$\overline{1}$ http://www.oru.se/ExCITE/.

2 http://www.giraff.org.
} 
Fig. 1 The GIRAFF robot
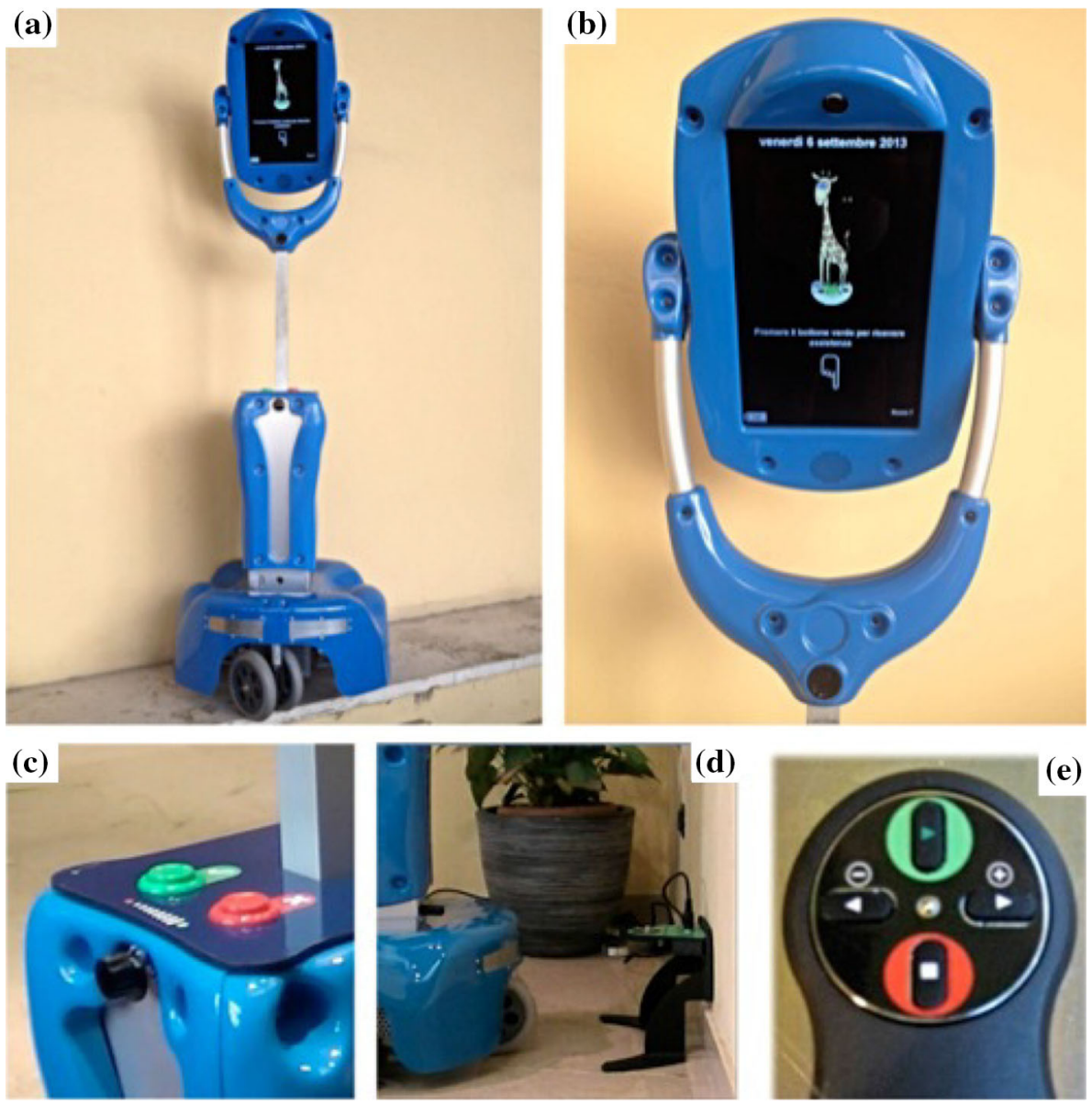

Secondary users can leverage the following functionalities of the robot: performing videoconference calls with the primary user at home; driving the robot, navigating and virtually visiting the environment; moving the tilt of the robot changing the point of view of the webcam, i.e., observing from different perspectives the primary user and the status of the environment; taking direct control of the robot in case of emergency $^{3}$. Alternatively, a primary user can initiate a call with a secondary user who is designated as a primary contact for him/her.

Overall, even though its functionalities are rather simple, the robot offers an augmented communication channel between the primary and secondary users that can be exploited to foster socialization, facilitate contacts as well as increase the opportunities of interaction.

\footnotetext{
${ }^{3}$ In general, when a secondary user calls the robot, primary users can accept or decline a call, as a regular phone call. In case of emergency, secondary users with proper authorization, e.g., a son of the old person, are able to take control of the robot without any action from the primary user. This modality has been included to allow access to the robot when primary users are not able to respond, e.g., because of a loss of consciousness.
}

\section{Related Works}

Previous research in Human-Robot Interaction (HRI) emphasizes that a prolonged interaction between a user and a robot can influence the attitude and behavior of the person and thus the user experience and the relationship with the adopted aid [14-21]. An example of longitudinal study is the one related to the PARO robot, a seal-shaped robot endowed with touch sensors, able to respond to external stimuli and designed for therapeutic interventions with older people and children with genetic syndromes and developmental disorders. The effects of a continuous use of PARO have shown, for example, an improvement in the relationship ability and of the stress levels in elderly patients [22,23].

More recently, [24] described the results of an ethnographic study, in which an assistive robot interacted with 55 elderly residents in a nursing home, for a period of three months and a half. The robot was operated remotely to interact verbally with patients. The analysis of the interviews and the results of systematic observations suggested that the robot had been well accepted among residents, especially for its ability to engage socially patients by recalling their attention and starting simple conversations. Nevertheless, the effects 
of long-term interaction between a human user and a robot do not always have positive implications. It may happen that the initial effect of novelty and curiosity toward a robotic aid and its use, fades rapidly inducing a decline of personal interest and changes in the user attitude ([16,21,25-27]. In this light, longitudinal studies, although they may be organizationally difficult and expensive especially in ecological contexts, are increasingly more relevant to understand the key factors that can promote an effective durable interaction.

A recent review shows three longitudinal studies carried out in the home context in which adult participants [28,29] or younger elderly [30] interact, for periods of time ranging from a minimum of 10 days and a maximum of 10 months, with robotic aids designed for different purposes. What emerges from the results of these studies, is the central role of functional and practical aspects related to the use of an assistive robot, the robot's social skills and personal expectations. These three factors play a fundamental role in promoting long-term interactions and in fostering positive experiences.

Another important aspect to consider relates to the methods used to collect data. Quantitative measures, such as the number and duration of the interaction between the person and the robot, video recordings, observational studies are usually used in the field of HRI [16,19,22-25,29,31]. However, these measures may not be properly suited for investigations in the home, where the user's privacy should be protected and respected. To identify and combine the most suitable instruments for a long-term evaluation in the context of domestic (intimate and private) environments becomes extremely important and useful to "capture" the daily routine of the person who benefits from a robot in his/her home. A good example of a combination of different qualitative and quantitative techniques is provided in [28]. In order to understand how "Roomba" (the well-known domestic robot for cleaning floors), was accepted over time as part of the domestic routine of a person, the use of interviews was combined with other survey instruments both qualitative (such as checklist of activities), and quantitative as questionnaires. This method of data collection, allowed to grasp the different time phases (pre-adoption, adoption, adaptation, use, maintenance of the robotic aid) that characterize and influence the effects of long-term interaction between a human user and a robot introduced in the home. This work also identifies a period of two months as the "long-term" interaction between a human and a robot, so as to be able to observe the effects of time. In fact, often the "long-term" is defined on the basis on the number of hours or sessions spent interacting with a robot, with or without specifying the period of time in terms of days or months of continuous use [25,32,33]. This way of establishing "long-term" interaction, obviously can not apply to domestic living contexts, where the interaction is continuous in time, it is established by the will of the person who benefits from a robot and entails the sharing of physical and social spaces that could help to promote or hinder the "long-term" interaction itself.

In line with this literature this paper presents a research design that aims to help assessing the long-term experience of telepresence robots. Specifically the paper introduces the MARTA Multidimensional Assessment of telepresence RoboT for older Adults, methodology that specifies: (a) the main aspects (i.e., variables) that in our view need to be considered in the long-term study of the user experience, and (b) the long-term evaluation administration (or timeline) that relies on the combination of mixed methods comprising qualitative and quantitative instruments administered over time. The paper also introduces the protocol for the deployment of the technology and its maintenance. The overall approach has been then tested within a complete long-term ecological case study of a telepresence robot deployment for one year assessment in a real home.

\section{Methodological Aspects for a Long-Term Evaluation}

The long-term evaluation of technological solutions inherently brings two fundamental problems that require to be taken into account: on one hand the technology should be installed, updated and maintained in an efficient manner so that no major technical problems emerge during the longterm experience that could negatively bias the evaluation; on the other hand the user perspective and systematic data gathering should be managed along the long-term exposure to the technology. This twofold perspective entails methodological aspects that are to be considered in the long-term evaluation. In this section we consider both problems and propose a combined methodological approach. More specifically Sect. 4.1 introduces our idea of a protocol for the technology deployment and maintenance in the long term, while Sect. 4.2 introduces a methodology for the evaluation of the user experience.

\subsection{Technology Deployment in Long-Term Test Sites}

When performing long term experiments with generic technological devices and, more specifically, with telepresence robots, one aspect particularly important is the need of having a robust working technology during the whole experiment so that the evaluation results are not biased by possible technical difficulties and malfunctioning. In this respect, we have defined and applied a test site deployment protocol that has the twofold objective of (a) providing guidelines related to a set of technical steps to be applied for successfully implement the test site; (b) identifying a set of relevant actors involved in the site management and their responsibilities so that any 
problem is promptly solved by the appropriate person in due time.

The relevant actors and respective responsibilities can be summarized as follows: a system administrator, is in charge of (1) managing the hardware configuration of the telepresence robot and (2) performing maintenance and major recovering activities; a robot administrator, is in charge of (3) managing and monitoring the remote users access to the robot, (4) supervising the functioning of the robot during its usage and (5) interacting with the system administrator to report technical issues; a test site engineer, is in charge of (6) implementing all the physical actions and (7) regularly visit the test site reporting to the robot administrator all the local issues. It is also worth underscoring how the system administrator and the robot administrator can be involved in supervising more than one test site/robot.

Figure 2 summarizes the proposed deployment procedure as it has been used during the EXCITE project. The procedure is composed by four different phases: (i) Setup, to configure the test site; (ii) Installation, to physically deploy and activate the telepresence system in the house of the primary user; (iii) Execution and Maintenance, to continuously supervise the test site activities and, in case of need, to provide suitable technical interventions; (iv) Closure, to remove the robot and shut-down all the associated services. Each phase requires some instrumental technical steps to properly contribute to the experiment correct realisation.

To give a feeling of the task included in each of the steps here is a short description. The setting of a generic GIRAFF robot test site is considered as the target. The Setup phase can be implemented in a relatively simple way: an internet connection is activated in the house by the test site engineers in collaboration with the end users (in case there is one already no activity is needed). The system administrator mainly synthesizes the robot configuration. It is worth saying that the users' registration and the access definition are performed by the robot administrator through an administration web service which is a back-end service provided by the robot company. The Installation phase consists in the

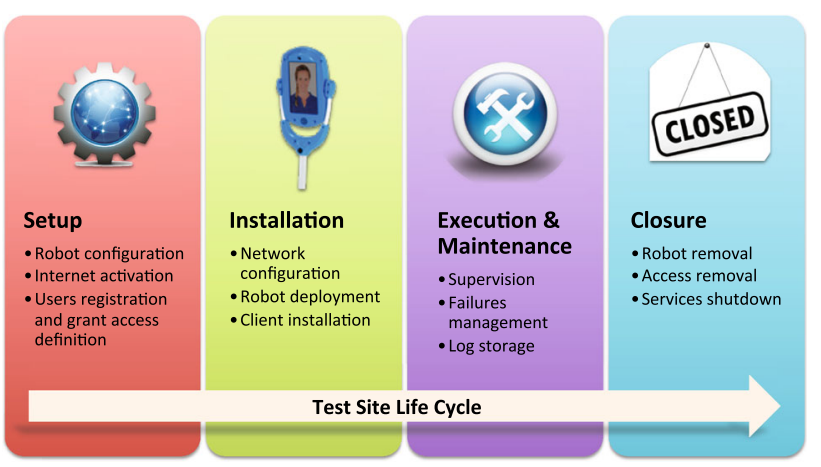

Fig. 2 Procedure for long-term test sites deployment test site engineer performing both the network configuration and the physical robot deployment. Configuring the network entails the setup of a WiFi router according to specifications provided by the robot company. The client installation is a simple task that just requires the secondary users to download a software from the GIRAFF web site. The Execution and Maintenance phase is clearly the one requiring the major effort as several technical not-planned issues may occur on the robot. The robot administrator is supposed to perform supervision and is responsible for failures management. Once the robot administrator detects problems by monitoring the activities (or if contacted by the end users) can ask the engineer for a technical intervention. Usually, once a problem is detected, the engineer and the robot administrator interact to figure out the possible causes of the problem. Then, two possible situations can be reached: either the test site engineer can locally manage the issue or a major intervention by the system administration is required. In the first case, the engineer has to visit the test site and try the proper recovery actions. Otherwise, the robot administrator informs the system administrator of the problem asking for suitable instructions. The last phase, the Closure of the test site, entails the robot removal by the test site engineer while the robot administration will perform access removal and the more general services shutdown by means of the administration web service provided by the robot company.

The methodology described here is functional to a proper evaluation of the long-term interaction since the latter could be inevitably influenced by problems occurred in one of the mentioned phases. To this purpose the technical team's role is to make the overall management smooth and to prevent the occurrence of problems.

\subsection{MARTA: Multidimensional Assessment of telepresence Robot for older Adults methodology}

The complementary aspect of the long-term evaluation is to observe the user experience in time and gather data on the interaction. To this purpose we introduce the Multidimensional Assessment of telepresence RoboTfor older Adults, (MARTA) methodology. MARTA defines: (a) our selection of the main variables of interest in the Human-Robot Interaction of telepresence robots and users within the domain of assistance of elderly people with the emphasis on the ability of the robot to act as an aid to support the social interaction; (b) the long-term evaluation administration (or timeline) that relies on the combination of mixed methods comprising qualitative and quantitative instruments administered over time. More specifically, Section 4.2.1 introduces the selected set of variables also mentioning the various instruments that can be used to assess them while Sect. 4.2.2 describes the administration timeline for these variables that specifies a possible plan to measure variables' changes over time. 


\subsubsection{The MARTA Components}

In order to define the main components that can influence the long-term assessment of telepresence robots able to support older persons, it is worth highlighting the fact that different typologies of users should be considered. More specifically the assistive domain for supporting elderly people entails the need of considering different users of the telepresence system that can be subdivided according to two different categories:

- Primary users, i.e., the older adults living in the home environment and hosting the telepresence robot;

- Secondary users, i.e., the users connecting to the robot in the house of the older adults and that can be in turn subdivided into: (a) professional caregivers belonging to a Health care organization; (b) family members (in the role of caregivers); (c) other relatives or friends who may visit the elderly person through the robot mainly for social purposes.

The different typology of users entails the need of focusing on different aspects connected to the evaluation. For this reason we identified a set of variables of interest that cover a variety of aspects involved in the long-term assessment of the telepresence robots.

The different variables can be combined according to the needs of the study as will be explained later in the subsequent section.

Table 1 summarizes the main variables that have been taken into account to evaluate the telepresence robot and its interaction with the users. More specifically the considered variables are grouped into three main categories:

- Screening measures, i.e., a set of variables useful to have the selection of participants and any general information about them;

- Social-Health and Psychological Measures, i.e., a set of variables useful to have a comprehensive idea of the users from a psychological point of view and with respect to his/her perception about socio-health status;

- Technology Impact Measures, i.e., a set of measures related to the impact of the technology in the humanrobot interaction and more specifically on the users' daily life.

For each of these variables Table 1 shows the reference to the inventory that can be used to assess them, and indications about the possible use of qualitative approach to measure it, a short description and also an indication about the typology of users that can be of interest for the given variable. This table constitutes our proposal for a reference system describing the interesting components in the Human-Robot Interaction with telepresence robots. It could also be expanded with potential additional measures in case of particular needs.

\subsubsection{The MARTA Administration Timeline}

The MARTA components described in the previous section introduced the main variables to be considered in an evaluation of telepresence robots and their interaction with older adults. A key issue of the evaluation is also to assess the process of adoption and effective use of a technological solution over the time so as to explore the effects of habituation or possible reasons of rejection by end user.

In fact, in order to assess the human-robot interaction it is important to assume a longitudinal perspective and understand how people interact with robots over time. In this respect the methodology proposed in this paper is based on the idea of using a method that allows to evaluate features over time.

Figure 3 gives the intuition of the general idea and how the MARTA methodology can be administered. Generally speaking, the evaluation is conceived as composed of a period of $N$ months during which a primary user has the robot at home and the secondary users can visit him/her through it. The assessment of specific variables happens at milestones $T_{i}$. Specifically, after an initial assessment $\left(T_{0}\right.$ in Fig. 3) at the beginning of the experimentation (baseline), some variables of interest are chosen among the ones belonging to the MARTA components (see Sect. 4.2.1) and are assessed at regular intervals $\left(T_{i}\right.$ with $\left.1 \leq i \leq n\right)$ including a final step $\left(T_{n}\right)$ to observe changes over time. After a period of usage the telepresence robot is removed from the end user apartment and the same variables will be assessed again after some months from this removal $\left(T_{n+i}\right)$.

Typically the screening measures will be used at time $T_{0}$, while the Social-Health and Psychological Measures and Technology Impact Measures can be iteratively used along the evaluation phases so as to observe potential changes over time.

The selection of the different measures belonging to these last two categories may depend on the specificity of the test site (or case study). Additionally, a combination of quantitative and qualitative instruments (questionnaires and interviews/diaries) can be used that better reflect the users needs and preferences as reported in Table 1.

It is worth observing that the benefit of the subdivision of the evaluation into phases is twofold: on one hand it allows capturing data in different "salient" moments of the user experience, on the other hand it allows synchronizing the results' interpretation with the technological life of the system, by looking at the reports from technicians and their monitoring of the test site life with respect to the technology 
Table 1 Components of the Multidimensional Assessment of telepresence RoboT for older Adults system

Telepresence Robots Assessment system

Multidimensional Assessment of telepresence RoboT for older Adults-MARTA system

\begin{tabular}{|c|c|c|}
\hline Measure & Function & User \\
\hline \multicolumn{3}{|l|}{ Screening Measures } \\
\hline Socio-Demographics & $\begin{array}{l}\text { Provides general demographics about a person to } \\
\text { include in the study like age, education, familiarity } \\
\text { with technology, etc. }\end{array}$ & Primary users \\
\hline \multicolumn{3}{|c|}{ Social-Health and Psychological Measures } \\
\hline $\begin{array}{l}\text { Perceived Loneliness-[34] } \\
\text { Loneliness Scale }\end{array}$ & $\begin{array}{l}\text { The UCLA Loneliness scale assesses subjective feelings } \\
\text { of loneliness or social isolation }\end{array}$ & Primary users \\
\hline $\begin{array}{l}\text { Perceived Health Status-Short } \\
\text { Form Health Survey (SF12) [35] }\end{array}$ & $\begin{array}{l}\text { The SF1 } 2 \text { measures how a person perceives his/her } \\
\text { health status }\end{array}$ & Primary users \\
\hline $\begin{array}{l}\text { Perceived Social } \\
\text { support-Multidimensional }\end{array}$ & $\begin{array}{l}\text { The MSPSS measures how a person perceives the social } \\
\text { support }\end{array}$ & Primary users \\
\hline
\end{tabular}

Scale of Perceived Social

Support [36]

Perceived Health

Support-Health Service

Satisfaction Inventory

Depression-Geriatric

Depression Scale [37]

Positive Affect Negative Affect

Scale-PANAS, [38]

support

A modified version of the Health Service Satisfaction Inventory

Primary users

the GDS is usually used to assess depression symptoms in the elderly

The PANAS measures the extent to which the affect (positive or negative) is experienced in a specified time frame or experience

Technology Impact Measures

Usability-System Usability Scale (SUS) [39]

To assess the usability of the software used to operate the telepresence robot; Additional questionnaires can

Acceptance-ALMERE model be used to adapt to the specific case of interface for tele operated robots

The Almere model can be used to test older adults' acceptance of assistive social robots

[40], interview

The PIADS assesses the psycho-social impact of the technological device on the users' life

Assisted Device -PIADS [41]

Telepresence-Temple Presence

The TPI is a tool to measure dimensions of (tele)presence Inventory (TPI)[42]

Support Expectation (and Assessment)—An ad hoc questionnaire, interview, periodic diary

This questionnaire measures the users' expectations (and assessment after a period of usage) on the ability of the telepresence robot to support the communication/monitoring between primary and secondary users. Slightly different versions of this questionnaire have been developed to make it suitable to the different typology of users (formal vs. informal caregivers or friends)

Attitude-An ad hoc questionnaire [3]

This questionnaire measures the users attitude with respect to the robotic aid within the domestic environment in terms of Intrusion, Advantages, Mistrust, Difficulty in management and Satisfaction

Secondary users

Primary users

Primary users, Secondary users

Primary users, secondary users

Primary users, secondary users

Primary users, secondary users

Primary users, secondary users

Primary users, secondary users functioning. In fact, the combination of data gathered from both the technological point of view and the user experience point of view allows a deeper and more informative analysis of the results.

In the next section we provide the description and complete data analysis performed on a real example of case study performed through the described approach.

\section{An Ecological Long-Term Case Study}

To demonstrate the implementation of the MARTA methodology and study the impact of a telepresence robot, this section describes an ecological long-term case study.

The main motivation for this study was to understand how the use of the telepresence robot and the interaction through 
it between primary and secondary users is influenced by time and long-term experience. Specifically we aimed to understand the user's experience, attitude, interaction behaviors, acceptance and beliefs towards a technological solution and how they impact on the adoption and effective daily use of a telepresence robot in a real context of daily life. Additionally we wanted to understand which are the technological challenges that need to be faced for a real and successful deployment of this new generation of supportive robots.

\subsection{Participants}

The participant's sampling strategy was based on a "combination or mixed purposeful sampling" method that combined "convenience sampling" and "chain sampling" methods [43]. Participants have been then recruited on a voluntary basis, exploiting contacts with potential users and healthcare professionals operating in the territory. The inclusion criteria were no memory failure or trouble in remembering their routines. Finally, a preference was given to those persons whose physical limitations could have a negative influence on their opportunity to have social contacts. The final choice was to involve a couple of older adults as primary users of the telepresence robot (see Fig. 4, left and central picture).

The woman is 84 years old. She has problems with her sight; she has no experience with technology in general, apart the television, and never uses computer in everyday life.

The man is 86 years old. He has reduced mobility and, like his wife, never uses technology in everyday life apart the television. They are quite independent although they suf-

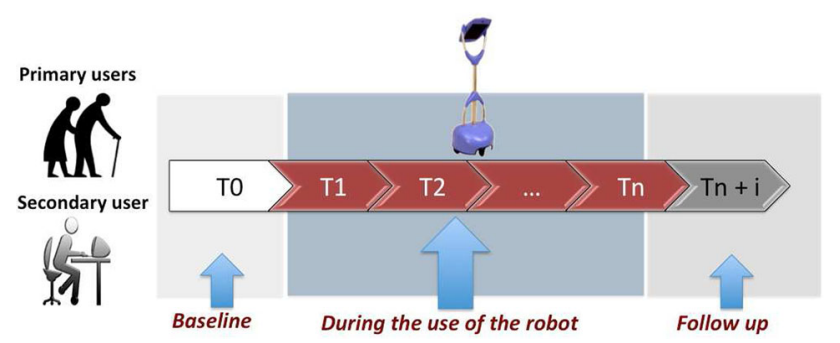

Fig. 3 Timeline for Telepresence Robots Assessment methodology for administering MARTA components fer from deterioration in their overall health status. They live in a country near Rome and spend all the time in their home having difficulties in going outside. Their social network with the external world is rather limited. They accepted to participate in this study to communicate mainly with their son, who is the secondary user of the system.

The man (Fig. 4, right picture) is a very busy person who lives in Rome ( $25 \mathrm{Km}$ far from his parents) and visits them on a regular basis (usually once-twice a week). He is 55 years old and has a high experience with technology in general using most of the technological devices daily both at work and for personal reasons.

\subsection{Tailoring the MARTA Methodology}

The methodology used for this assessment is based on mixed methods approach comprising qualitative and quantitative instruments for the long-term assessment and specifically on the MARTA methodology. The main components chosen for this study were the following: for the primary users we considered the technology acceptance, attitude, affective response to technology, impact in everyday life. For the secondary users we chose expectations, usability, spatial presence, affective response to technology and impact in everyday life. In addition we were also interested in observing the frequency of usage of this technology and the overall assessment of the experience on behalf of both type of users.

The general evaluation described in the previous sections has been implemented and adapted to satisfy the needs of this specific case study. Figure 5 describes the procedure we used for this test site. The entire evaluation session lasted $\mathbf{1}$ year from January 2012 to January 2013. Specifically, at the beginning of January 2012 (T0, Setup and Installation phases of the test site) we installed the robot in the house of the old people couple and the pilot software on the portable computer of the secondary users. From January 2012 to December 2012 the old couple and their son had the possibility to communicate through the telepresence robot $(T 1, T 2$, Execution \& Maintenance phase). At the end of December 2012 (T3, Closure phase) the robot was removed from the apartment of the old couple. In January 2013 (T4) a follow up assess-
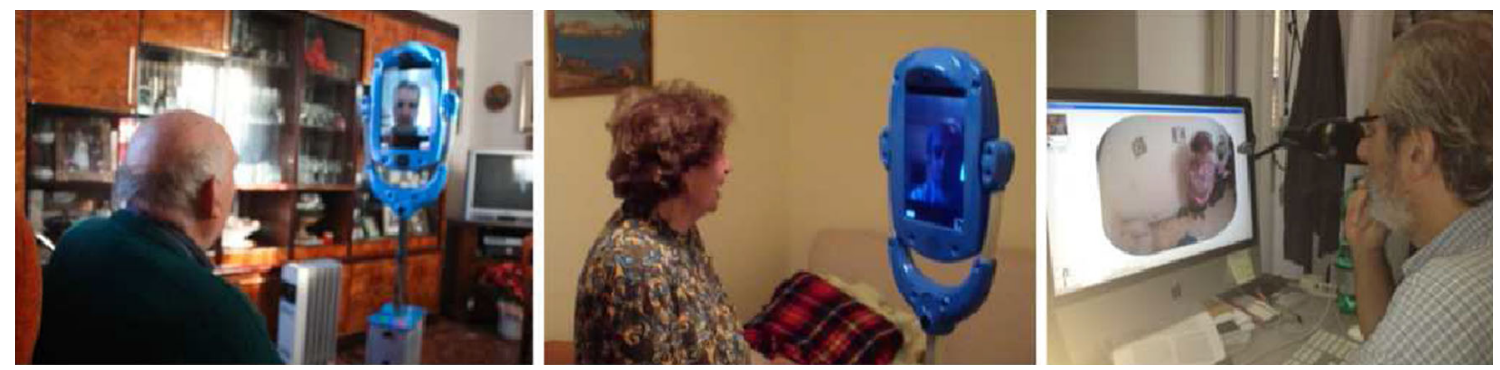

Fig. 4 Primary and Secondary users of the long-term case study 


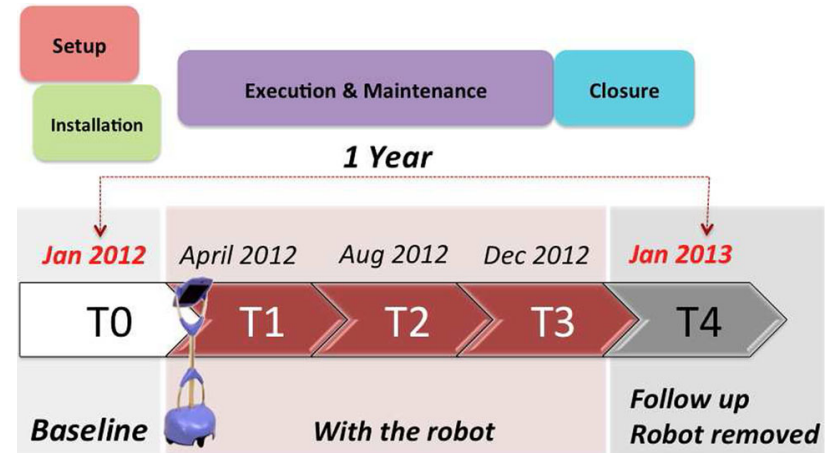

Fig. 5 The Long-Term Evaluation procedure

ment has been made to understand the effect of the robot's removal. During the whole experience, every 4 months we checked some specific variables chosen from the MARTA components administrating to both primary and secondary users specific questionnaires and interviews as follows:

\subsubsection{Primary Users}

T0: At time $T 0$ we asked the primary users to fill in the consent form and the Socio-Demographics Data Form and the Almere Acceptance Model. The administration of additional questionnaires related to the Socio-Health Perception and Psychological Measures was not possible due to the specific conditions of the couple.

$T$ 1: After a period of usage at time $T 1$ the primary users filled in the Attitude questionnaire that studies the individual attitude with respect to the robotic aid within the domestic environment.

T2: At time $T 2$ we asked the primary users to fill in the Temple Presence Inventory and the Almere Acceptance Model questionnaires.

T3: At the end of the overall experience we administrated to the couple the PANAS and PIADS questionnaires, the Almere Acceptance Model, the Attitude questionnaire and a final interview.

T4: After the removal of the robot from the couple's apartment we interviewed again the primary users to assess the effects of the absence of the robot.

\subsubsection{Secondary User}

T0: At time $T 0$ the secondary user filled in the consent form, the Socio-Demographics Data Form, the Support_Expectation questionnaire and the periodic diary.

$T$ 1: After a period of usage at time $T 1$ the secondary users filled in the Usability questionnaire and the periodic diary.

$T 2$ : At time $T 2$ we asked the secondary users to fill in the Temple Presence Inventory, the questionnaire for Sup-
port_Assessment, the Usability questionnaire and the periodic diary.

T3: At the end of the overall experience we administrated to the secondary user the PANAS and PIADS questionnaires and a final interview.

T4: After the removal of the robot from the couple's apartment we interviewed again the secondary user and administered the Support_Assessment questionnaire to assess the effect of the absence of the robot.

During the whole period the frequency and the durations of the calls made through the robot were also registered to examine possible changes in the frequency of usage over time.

\subsection{Results}

\subsubsection{Primary Users Results}

Technology Acceptance Mean scores of selected constructs of the Almere model have been calculated to access the level of acceptance of the robot on behalf of primary users over time (see Figs. 6, 7).

Overall, the telepresence robot emerged to be positively evaluated by both the Male and Female user with respect to Perceived Usefulness (PU), Intention To Use (ITU) and Attitude (ATT) that maintained high scores along the three phases $T 0, T 2$ and $T 3$. More specifically for both users the Intention to Use, ITU, the Perceived Usefulness (PU) as well as the Perceived ADaptiveness (PAD) of the system increased over time. Both primary users stated that they would be willing to continue using of the robot showing a positive attitude towards GIRAFF.

In the pre-adoption phase $T 0$ a level of ANXiety (ANX) has been perceived by the female user $(M=5)$ in comparison with the Male user $(M=3)$. The perceived anxiety diminished along time, even though for the female is maintained at a rather high level $(M=4$ at $T 3)$ with respect to the Male user $(M=2$ at $T 3)$. It is worth highlighting that the Female primary user was indeed the one responsible for the robot maintenance in terms of attention, control of the functioning and probably this aspect induced more anxiety on her with respect to the male primary user.

The Perceived Ease Of Use (PEOU) of the Female user is rather high along time, while it slightly diminishes for the Male primary user who somehow had a more "passive role".

For the other constructs the two primary users showed similar scores indicating moderate Perceived ENJoyment (PENJ), over time and, sufficient perceived Facilitating Conditions $(F C)$. The individual perception of Social Influence $(S I)$, is rather low for both primary users maybe because their son remained the only one to contact them through the robot. 
Fig. 6 Results from the Almere technology acceptance model along $T 0, T 2$ and $T 3$ for the Male participant: Means (scale $1-5)$

Fig. 7 Results from the Almere technology acceptance model along $T 0, T 2$ and $T 3$ for the Female participant: Means (scale 1-5)
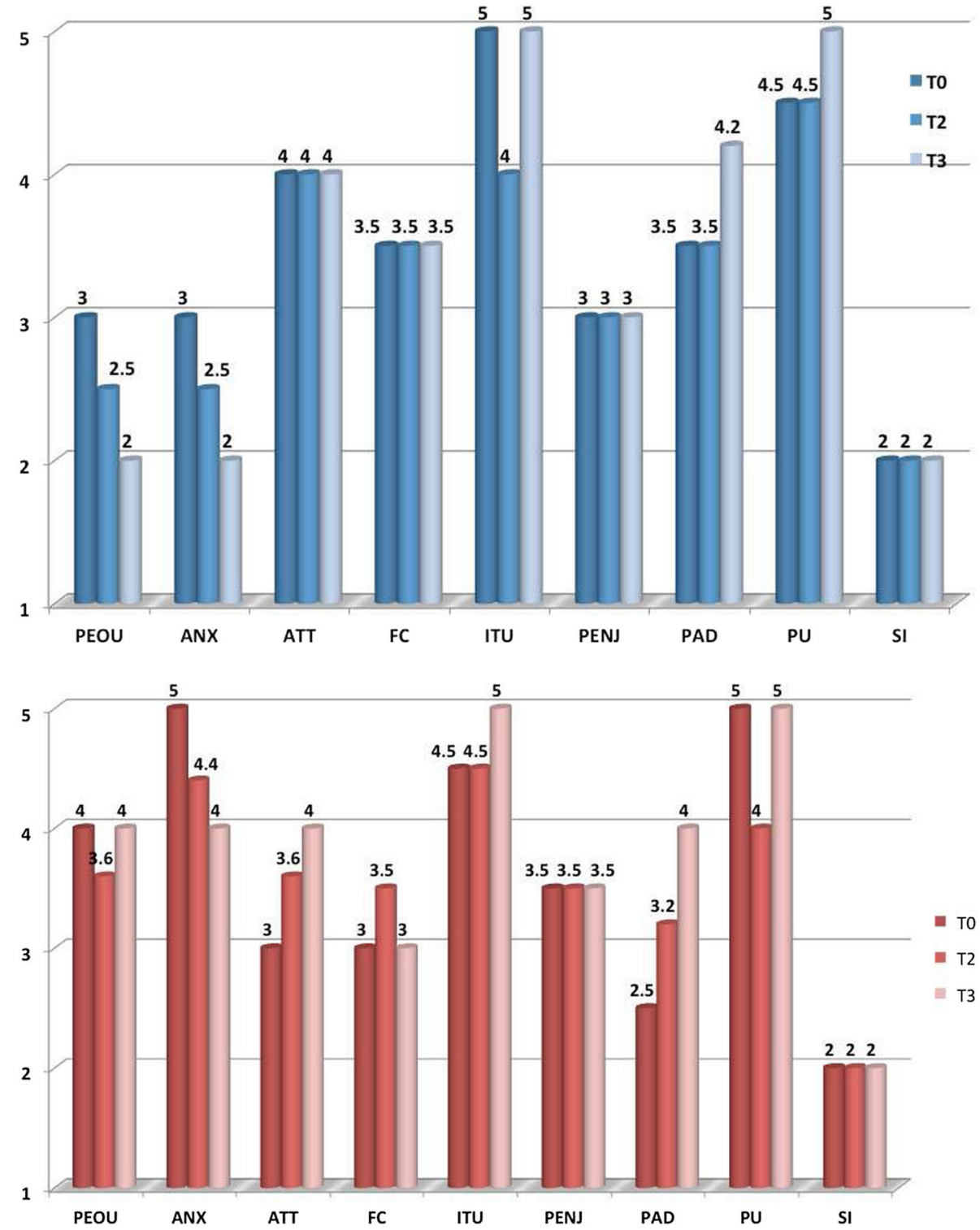

For both users, the robot is not perceived as an ele-

Telepresence Dimensions This paragraph reports the analysis of the distribution of responses related to the $a d$ hoc questionnaire adapted on the basis of the Temple Presence Inventory and the Networked Minds Social Presence Inventory. It is worth emphasizing that not all the survey questions were applicable to primary users, because some of them were judged as too difficult to understand. The dimensions which clearly express the point of view of the primary users are shown in Fig. 8. The quality of the interaction, and the social presence were deemed satisfactory by both users. A little bit less satisfactory is the perception of copresence and perceived psychological involvement for both users.

Attitude Figure 9 shows the mean scores of the dimensions related to the Attitude questionnaire adapted from [3]. ment of intrusion into their home life even if this perception experiences a slight increase in the $T 3$ phase, nevertheless remaining at a low level. The benefits related to the use and presence of the robot at home are valued and their recognition is maintained over time. There is a good satisfaction with the functions and features of the robot. Users do not have an attitude of distrust (in terms of psychological distance) of the robotic aid. Finally there is a difference between the male and female about the concerns relating to the management and maintenance of the robot, which increases over time for the female user.

Affective response Primary end users reported a rather positive affective response towards their experience with the telepresence robot. Specifically, the score of Positive Affects on PANAS scale was respectively 41 vs 19 (on the Nega- 
Fig. 8 Results from the Telepresence questionnaire for both the Male and Female Primary User: Means (scale 1-7)

Fig. 9 Results from the Attitude questionnaire for both the Male and Female Primary User at Time T1 and T3 respectively: Means (scale 0-4)
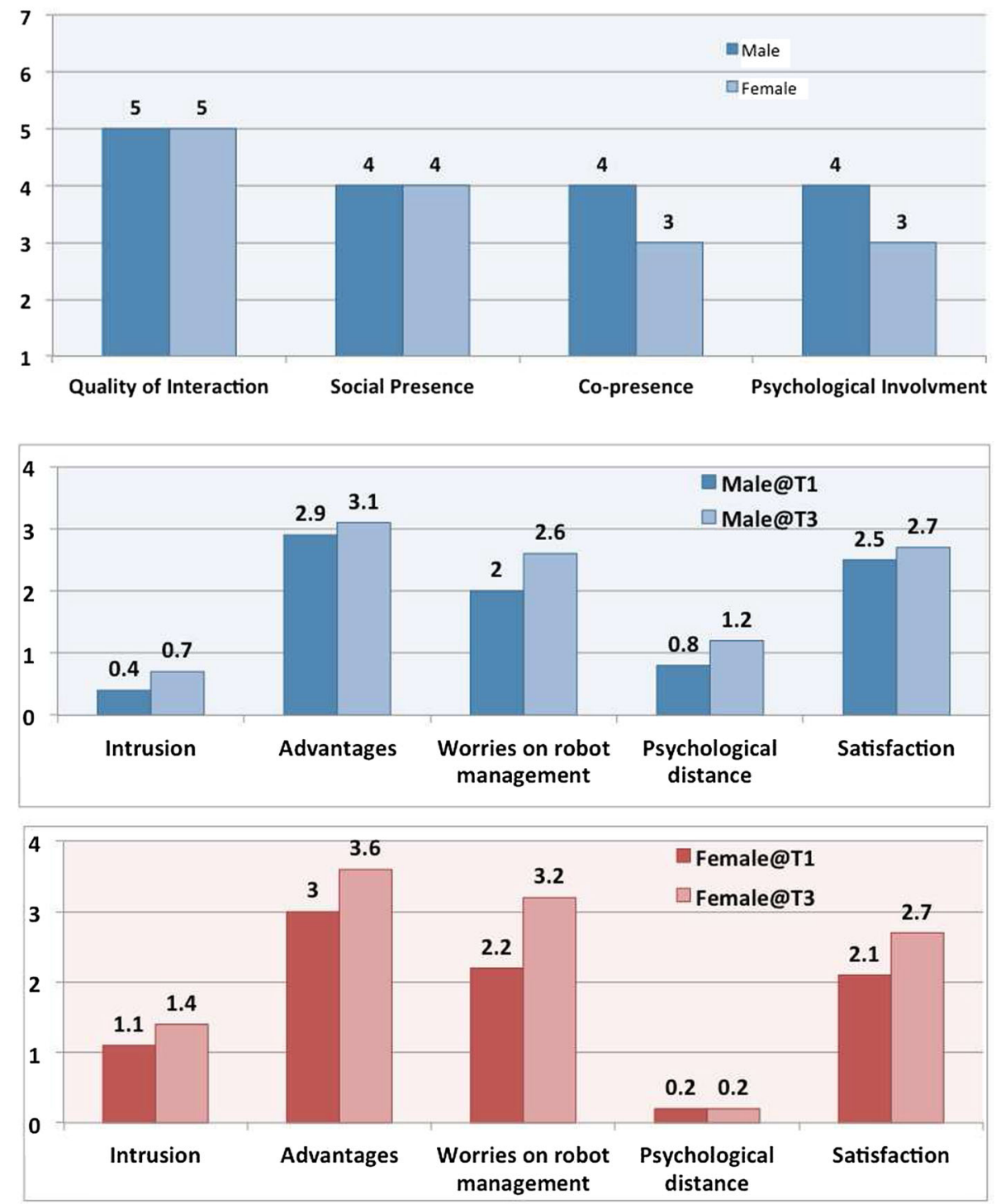

tive effect sub scale) for the female and 38 vs 21 (on the Negative effect sub scale) for male primary user. Scores of both users are indicative of a positive emotional valence with respect to the experience of use and interaction with the robot. In addition, the emotional reaction measured by the affective scale of our Attitude questionnaire, showed that primary users affectively responded in a very similar way to the robot. Specifically, a high scoring was found on the positive adjectives fun, interesting, useful, stimulating and reassuring.

Impact on everyday life Considering female primary user's perspective the robot did not have a strong psychosocial impact on her life. Comparing the scores of the three subscales of the PIADS questionnaires it is possible to note a small improvement in the ability item $(M=0.83)$ with a significant increase in the sense of independence and adequacy in dealing with the situation of daily life associated with the use of the robot. A positive impact is also obtained for the self-esteem subscale $(M=1)$ where, in particular the use of the robot had a positive effect on self-esteem and security. The use of the robot did not have any effect in terms of adaptability.

With respect to the male primary user, using of robots had a positive influence on adaptability $(M=1.5)$ and in particular the sense of well-being, ability to participate, eagerness to try new experience and adaptation in activities of daily living. A minimum improvement due to the use of the robot was also related to self-esteem $(M=0.75)$ with specific reference to the sense of confidence and safety. No real improvement in terms of ability has been found.

Overall assessment The semi-structured interview administered to the older users was aimed to investigate the opinions of the couple on the long-term experience of use and on the effect of the removal of the robot at the end of the experiment. 
The interviews were transcribed verbatim and analyzed by a first researcher who identified and organized a series of main topics and relevant end users' responses from transcripts of the Interviews. A first coding system was developed to classify the end users' responses emerged by the transcriptions. Subsequently, a second researcher coded the end users' responses of the transcriptions. As a result of a comparison between the results obtained by the two researchers and a complete agreement obtained on the coding of the interviews the most appropriate code was established.

Overall, the robot has been appreciated for its ability to create company and its integration in the home environment. The older users were satisfied with the overall experience. There were no particular disadvantages associated with the use of the robot in the entire year of assessment. Expectations of use also seem to have been met. Both users declared, also after the follow-up phase, that would be willing to continue to use the robot. Some negative comments and suggestions also emerged. In the following we describe some more detailed considerations organized by categories that support the judgments of the overall experience.

- The robot as a companion and support to loneliness One of the interesting observations from the interview is that according to both primary users the robot has helped to keep them company and to alleviate the sense of loneliness. This result is in line with the basic idea of the project within which the experiment is inserted, i.e., using telepresence as a tool to alleviate social isolation and loneliness. Here are some comments to support this view: “.... I am happy when I hear it ringing because I think it is a company to us, ... we are often almost always alone, even if it is there doing nothing for us it is a company... ]"; [ " .... I agreed to participate in this study with the hope of being able to use the system and take advantage from it. But I do not think to it as an appliance, I think it is rather a company for a man who is getting older].

- The added value of the video and the movement $A$ positive factor of the communication based on the telepresence robot seems to be the possibility to visually see the calling person unlike for example the phone that allows only audio communication. [... It was very convenient that we could see our son]; [... to see the other is the liveliness of the device. You see the person, that is the added value, it is a great feeling, I was never bored....]. The ability of the robot to move in the environment has been also appreciated by primary users.

- Robot's appearance and its integration in the domestic environment The appearance of the robot was judged as quite pleasant as well as its integration in the home was assessed as smooth. Some advices on both of these factors, however, did emerged. The recommendations concern the possibility of having an adjustable height for the robot, both to better interact and to give to the robot a greater stability in the movement; furthermore, the fact that the robot is positioned with the screen in front of the walls when recharging was not appreciated because it would be more appropriate for them to have higher control on it when a call is incoming. Alternatives such as anthropomorphic robots or robots that resemble a pet have not been preferred to the current one. It was also mentioned the possibility to add manipulative ability to the robot as a possible added value.

- Difficulties in the management and anxiety for its maintenance Some difficulties on the management of the robot and anxiety related to its maintenance emerged. The management difficulties are mainly related to the fact of not being able to correctly reposition the robot to the charging base as this is an operation that primarily belongs to the secondary user: [... I'm afraid to touch it if it gets up from the docking station]; [... I'm anxious, I often go there to see if it works or not if it is okay or not. Then I think that it may be damaged when the power goes away ...].

- Usefulness The robot has been considered useful primarily for its ability to improve the contact and communication with the outside world and also in part for its ability to move freely in the environment. However, there are expectations of some missing features that would help a lot to increase the level of utility, which are mainly related to the safety of the house and of the person. [... I think it still will take a little time before it can be considered as really useful: let us for example assume that someone try to force a window to enter the house, the robot should communicate with the other robots or with the police. We are in a country, but for those who are in the countryside this could be of extreme help, the robot would be necessary]. ["... It should have the sufficient capacity to help a person. It must know what to do ... because the old person could loose the senses and the robot should be able to autonomously call for help "].

- Need for more control by the elderly The prolonged use in time of the robot has highlighted a need for greater control by the primary users. [“...I also would like to be able to move it. We call each other on the phone, do the numbers ... I would like to be able to do the same with the robot. There should be the keys with which you can call. I would like to control it"...]; ["there is a switch that can turn it off and turn it on, but I would like to have other buttons for other functions".]

The removal of the robot also caused a sense of loss in the primary users. In addition, users said they were willing to continue to use the robot. 
Fig. 10 Expectation (at $T 0$ ) of secondary user vs assessment after a period of usage (at T2)-Means (scale 0-4)

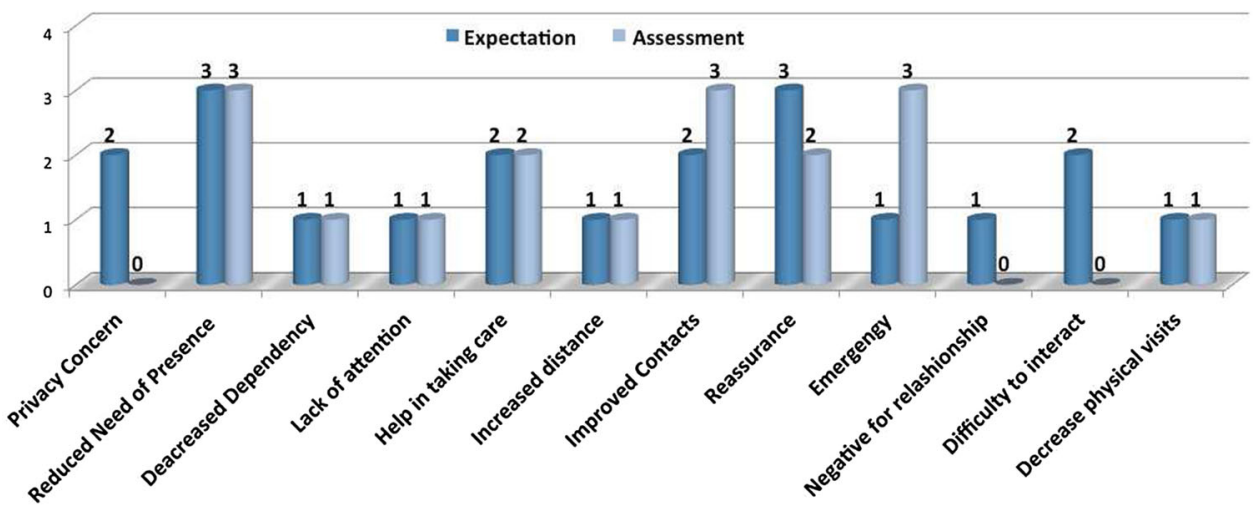

\subsubsection{Secondary Users Results}

In this subsection we describe the results for the secondary users. Data have been organized according to the following main categories which correspond to the variable of interest: Expectations, Usability, Spatial presence, Affective response and an additional category concerning the Overall Assessment of the entire experience. As last information we also report the frequency of usage over time during the entire one-year evaluation effort.

Expectation, assessment and follow up Figure 10 illustrates the comparison between the expectations of the secondary user on the system at the beginning of the experiment and the actual assessment after a consistent period of usage (at $T 2$ ).

In particular we can see how at the beginning of the experience the secondary user had shown a moderate concern for issues related to privacy $(M=2)$, which, after a period of usage is clearly reduced $(M=0)$.

The idea that GIRAFF may reduce the need for physical presence of the secondary user to support the activities of daily living is confirmed over time $(M=3$ for both the evaluation moments). The light perception that GIRAFF may contribute to make the primary users feel less dependent on secondary user is also maintained over time ( $M=1$ for both the evaluation moments). The concern that the communication through GIRAFF could be interpreted as a lower attention of secondary user towards their parents is low both in terms of expectations and assessment $(M=1$ for both the evaluation moments). A constant moderate feeling that GIRAFF is helpful to take better care of their parents is maintained ( $M=2$ in both the evaluation moments). There seems not to be concern that communication through GIRAFF can help to increase the psychological distance between primary and secondary users ( $M=1$ in both the evaluation moments). GIRAFF also seems to be helpful in maintaining a constant contacts, the expectation being largely fulfilled and growing over time (from $M=2$ to $M=3$ ).
The fact of feeling reassured when away from their parents is slightly decreasing over time (from $M=3$ to $M=2$ over time). The perception of secondary users that GIRAFF can be useful in unexpected situations or in case of emergencies grows over time (by $M=1$ for $M=3$ ). GIRAFF is not even perceived as a negative element in relation to the relationship between primary and secondary users (from $M=1$ to $M=$ $0)$.

The concern that secondary user might find it difficult to interact with his/her parents through GIRAFF $(M=2)$ disappears after a prolonged use of the system $(M=0)$. The concern that the use of GIRAFF may reduce the opportunities for physically visit their parents remains constant over time ( $M=1$ for both the evaluation moments).

Figure 11 shows the effects of the GIRAFF's removal from the elderly users apartment on the secondary users with respect to the variables introduced above. Specifically the pictures shows that the absence of GIRAFF after a period of usage did not particularly contributed to increase the sense of protected privacy $(M=1)$; the removal did not contribute to increase the impression of higher attention toward the primary users $(M=1)$, neither it has been perceived as a way to increase the closeness to the primary users $(M=1)$. However, the fact that the GIRAFF was not in the apartment anymore did not negatively influenced neither the relationship between primary and secondary users $(M=1)$, nor their ability to interact $(M=0)$. On the contrary the absence of GIRAFF did increased in the secondary user the feeling that the need of presence in his parent's home increased $(M=3)$; he also perceived that the GIRAFF's absence contributed to an increase in the dependence of their parents from him $(M=3)$; to an increase in the difficulty in taking care of his parents $(M=4)$; to an increase of the difficulty in maintaing frequent and constant contacts $(M=4)$; to an increase in the sense of worry and concerns when he is away from them $(M=4)$; to an increase in the difficulty to manage emergency situations $(M=3)$. On the contrary the absence of GIRAFF seems not to have a particular influence on the frequency of physical visits $(M=2)$. 
Fig. 11 Effect of the Giraff's removal (follow up at $T 4$ ) on the secondary users-Means (scale $0-4)$

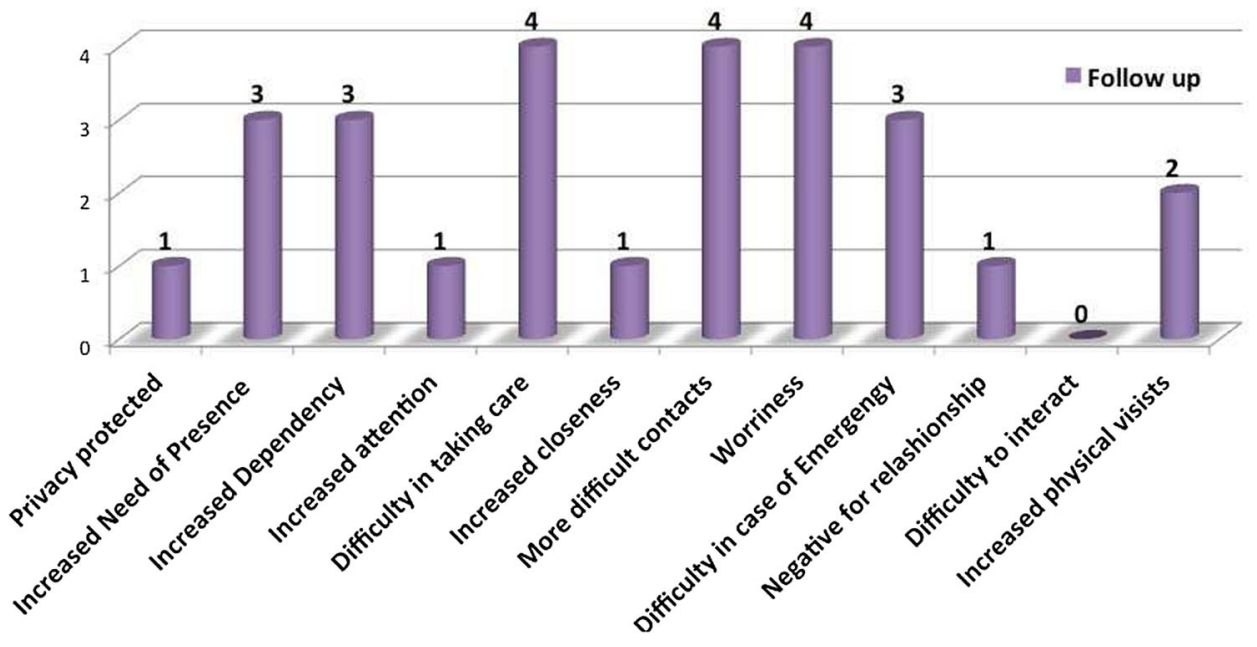

Usability The analysis of the SUS questionnaire showed a score of 52.5 indicating a low level of subjective usability of the GIRAFF's client interface. This result is also confirmed by the questionnaire on the usability of the system we developed ad hoc for the case study, which shows scores not particularly high. Figure 12 shows how the usability scores changed over time (from time $T 1$ to time $T 2$ ). Specifically, usability increased over time (total mean from $M=1.4$ at $T 1$ to $M=2.2$ at $T 2$ ) but some aspects of the usage remained difficult for specific activities.

Specifically the operations related to the docking procedure were judged as difficult. For example at time $T 1$ the following activities: "Go back to the docking station" $(M=0)$, "Position of the robot into the docking station" $(M=0)$ and "Know the battery status" $(M=1)$ resulted as particular difficult. The usability of these functions becomes a little better at time $T 2$.

Other aspects not reported in the figure also improved over time. Specifically the "Concepts and language of the computer application" became clearer and the user satisfaction of the robot's behavior increased. Overall, using the system for long period of time had a positive influence on the perceived usability that overall increased or remained constant.

Telepresence dimensions Regarding the telepresence dimensions (Fig. 13), the pilot user perceived an insufficient level of Spatial presence (sense of being physically located in a virtual environment) and Co-presence (psychological connection to and with another person). In addition, he had a moderate positive perception of Perceptual Realism (experience in which the mediated interaction accurately simulates or reproduces the sensory experience that would be expected in the non-mediated interaction), Social Presence (feeling of being together) and Perceived psychological engagement (extent to which the user feel mentally immersed in the experience). The experiences of Social richness (subjective experience of warmth and intimacy in the mediated Inter- action) and Perceived behavioral interdependence (extent to which a user's behavior affects and is affected by the interactant's behavior) are more satisfying.

Affective response The PANAS scale shows an average value of positive affect toward the technology that is relatively high but not significantly higher than the negative affect scale. We can deduce that there is a rather neutral affective response of the secondary user to the used technology.

Impact in everyday life The analysis on the PIADS scale reveals that overall the service offered through GIRAFF did not have a particularly strong psychosocial impact on the secondary user's life, not causing any remarkable changes. From the point of view of the three sub-scales of the PIADS it is possible to say that the main improvement has been obtained especially with respect to adaptability that is the predisposition of a person to take risks and try new experiences $(M=1)$, with a considerable increase in the capacity of participation and the ability to take advantage of situations.

The ability, that is the general skills of efficacy, obtained an average score of $(M=0.91)$, and results shows that in particular the robot has improved mainly the utility $(M=3)$. The independence, the ability and quality of life show an average score of $M=2$. The system has improved to a lesser extent the self-esteem, $(M=0.12)$.

Overall assessment The analysis of the interview to the secondary user highlights an overall positive opinion of the experience even though some shortcomings have also been mentioned.

\section{- The added value of the video and the movement} Overall, the secondary users appreciated the empathic communication supported by the robot "from the point of view of empathy this is an effective tool to chat...", "this object is interesting to perceive the environment around". The possibility of being able to make a video call and to 
Fig. 12 Usability assessment-Means (scale 0-4)

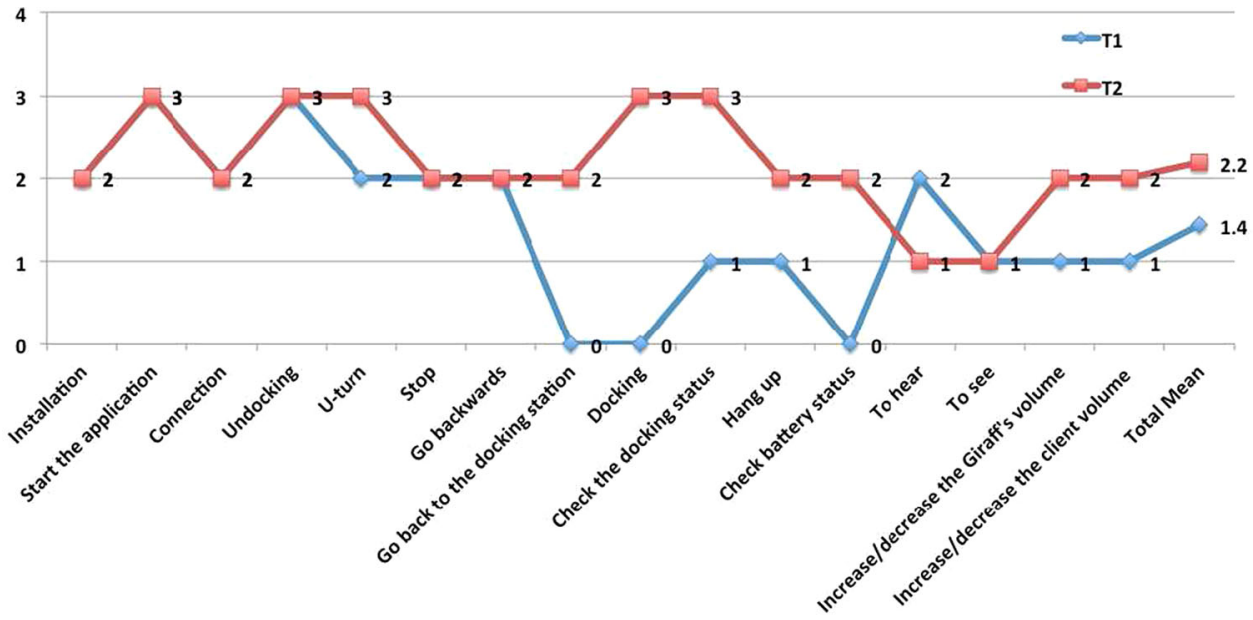

Fig. 13 Telepresence dimensions assessment-Means (scale 1-7)

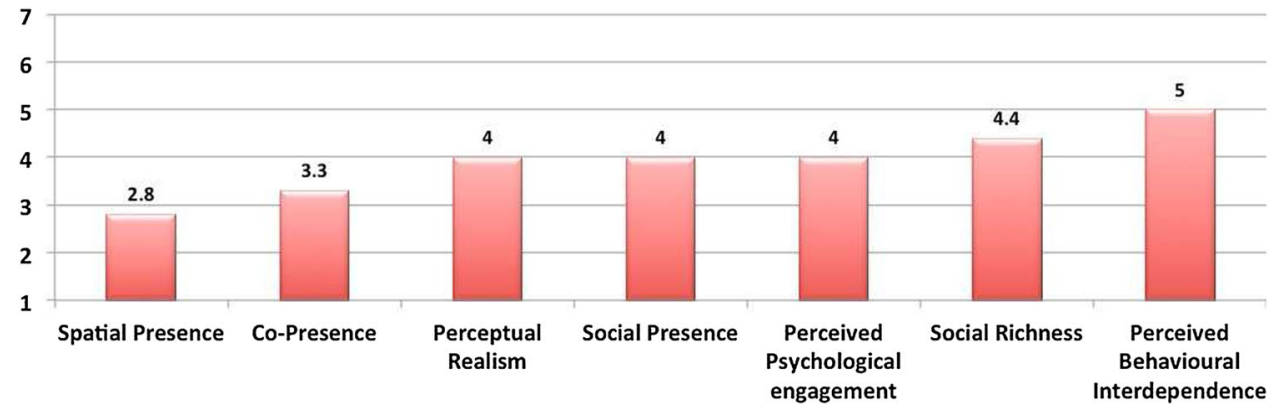

establish eye contact with his parents was one of the main advantages recognized by the secondary user, "I had the impression that connecting through the robot instead of using a phone, allows you to capture more, it is a way to establish a rapid human contact, and also my parents can see me".

The contact with the robot allows "to generate an effective exchange between people" more effective than a phone call.

Furthermore, the possibility of being able to "enter the environment and navigate through it" is considered a major advantage in that it "increases the sense of presence", "the fact that the robot adds context information contributes to a feeling of being at home which I never experienced before through a phone call".

- Anxiety for the system's maintenance and usability aspects

A particularly problematic issue that the user reported frequently during the interview, is related to the docking operation. This problem, especially in the early stages of use, had a very negative impact on the intention to use the system from the user: "at the beginning this difficulty was really a problem for me ... I was afraid to leave the robot in the middle of the room without a concrete possibility to go and move it away". The user had repeatedly expressed a sense of anxiety and effort in completing a successful docking operation. The fear of not being able to reposition the robot in the charging station had a negative impact on his intention to use the system to contact his parents. This anxiety and discomfort was mainly due to the health conditions of the primary users, "since my parents are fragile, I was worried to leave the robot in the middle of the room and this anxiety prevented me from calling them through the robot sometimes".

Despite some usability problems emerged, from the content analysis of the interview we can say that the telepresence system has been perceived as a communication device that allows to establish a valid empathic contact and closeness among real end users.

Frequency of usage During the period in which the test site was active, the total number of calls performed from both primary and secondary users has been 245. In Fig. 14, detailed information on the types of calls is depicted. In particular, Fig. 14a presents the calls according to the completion: the successfully completed calls were $73(30 \%), 17$ (7\%) calls were not answered, 39 (16\%) were declined, $106(43 \%)$ calls cancelled and $10(4 \%)$ calls have not been actually completed (i.e., an error occurred or there was a network problem). The total duration of the completed calls was more than $5.5 \mathrm{~h}$ (331.5 $\mathrm{min}$ ) and the average duration of a call was about 4.5 $\min$. 


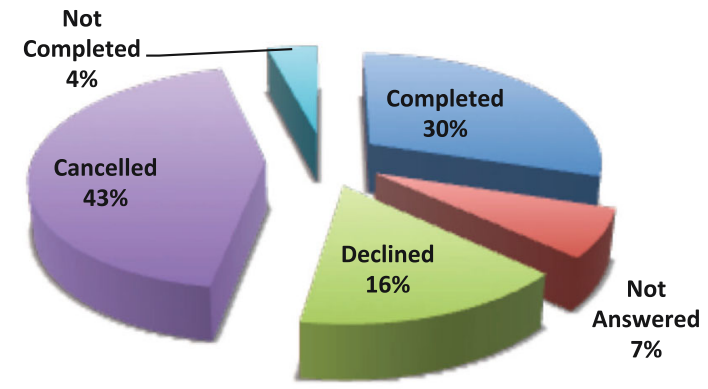

(a)

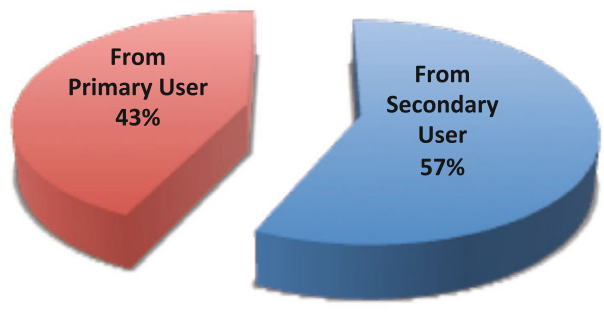

(b)

Fig. 14 a Total test site calls distinguished according to the type (i.e., completed, not answered, declined, cancelled and not completed). b Total calls distinguished by origins, i.e., calls from the secondary user and calls from the primary users

The calls were performed either from the secondary user to the robot (i.e., the secondary user was calling the robot) or from the robot to the secondary user (i.e., the primary users were calling the secondary user). Namely, the secondary user has performed $140(57 \%)$ calls while the primary users have raised 105 (43\%) calls (Fig. 14b).

Considering only the calls performed by the secondary user (see Fig. 15a), 54 (38 \%) calls have been successfully completed, 12 (8\%) calls received no answer, 36 (26\%) calls were declined, 33 (24\%) were cancelled and $5(4 \%)$ calls were not completed. The total duration of completed calls was more than $4.5 \mathrm{~h}$ ( $276.5 \mathrm{~min})$ and the average duration of a call was about $5 \mathrm{~min}$. As for the primary users calls (see Fig. 15b), 19 (18\%) calls have been correctly completed, $5(5 \%)$ calls have been not answered, $3(3 \%)$ calls have been declined, 73 (69\%) have been cancelled and $5(5 \%)$ calls have not been properly completed. The total duration of the completed calls was more than $50 \mathrm{~min}$ and the average duration of a call was about $3 \mathrm{~min}$.

The high number of cancelled and declined calls deserves an additional comment. In fact, this was mainly due to an initial low familiarity of both primary and secondary users in using the tool. In particular, the female primary user (i.e., the one mainly managing the calls with the robot) had difficulties in distinguishing the colors of the (green/red) buttons when starting/receiving a call. This has been more frequent in the first part of the experimentation when the user was learning

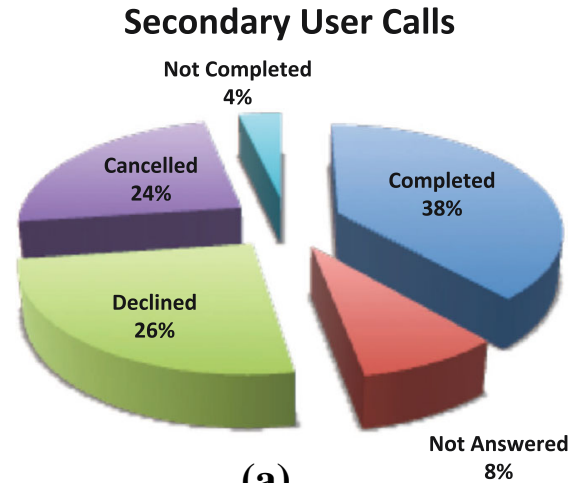

(a)

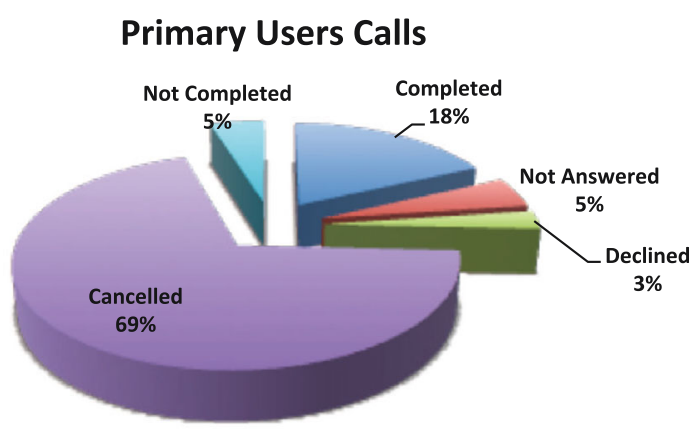

(b)

Fig. 15 a Secondary user calls and $\mathbf{b}$ Primary users calls distinguished according to the type (i.e., completed, not answered, declined, cancelled, not completed)

how to distinguish the functionalities of the buttons according to their position rather than according to their colors.

Then, after such initial phase, the users started to properly use the robot and on a more regular base. Namely, the average number of completed calls per month was 10 , and January and November 2012 resulted as the more intense months with 18 completed calls. The minimum number of calls has occurred in December 2012 with 3 calls (issued by the secondary user). Concerning separately primary and secondary users, the average number of completed calls per month by the secondary user was 7.7 spending almost $40 \mathrm{~min}$ per month in conversations through the robot. The month of November 2012 resulted as the more active one with 16 completed calls. The primary users performed 2.7 completed calls per month corresponding to almost 8 min conversations. The more active month was January 2012 with 11 completed calls.

\section{Discussion}

\subsection{Remarks on the Long-Term User Experience}

Overall, the long-term evaluation results are quite positive both for the primary and secondary users in terms of meeting 
their expectations, the functional and social acceptance of the robots and users attitude toward the telepresence robot. Contrarily to what is frequently found in the literature $[16,21$, 25-27], the effect of novelty and curiosity on the telepresence system did not have impact in the experience of use of this case study's participants. Specifically, the interest of users did not decrease in time.

Primary users The experience of long-term use of the two primary users reveals a consistently positive attitude over time in relation to the ability of the robot to act as a communication aid. Small differences between the female and male user emerged in particular in relation to the acceptance of technology and the attitude towards GIRAFF. In the preadoption phase, the female user showed a higher level of anxiety over the use of the robot. During the experience she had an increasingly active role in the use of GIRAFF feeling directly involved in the management and control of the operation of the robot. Yet over time acquiring greater familiarity with the robotic aid she continued to experience some anxiety. It is plausible hypothesize that some technical malfunctions during the assessment (linked mainly to internet connection problems and to some problems in answering the calls), have contributed to evoke similar feelings which, however, does not seem to have affected the perception of usefulness of the robot. The male user experienced a decrease over time of the perceived ease of use. This perception may be justified by a low sense of perceived psychological involvement and social presence. In addition, it is likely to think that because of his reduced mobility, the male primary may have experienced a less active role in interacting with the robot and observed the technical malfunctions and the resulting concerns of his wife interpreting them as a lower perceived ease of use compared to his initial idea. In general, we can say that both end users accepted GIRAFF in time and its presence in the house, showing a strong and prolonged intention to use the robot and perceiving the robot as a proper aid, very useful and beneficial in terms of their need to make eye contact-communication with their son and their feeling of loneliness, meant more in terms of social isolation, which was mainly reported from the male primary user. The use of the telepresence robot has had a more significant psychosocial impact on the quality of life of both the male primary user and the secondary user. The use of the telepresence system may have represented for the users, on one hand a new way to share experiences; on the other hand, for the secondary user it represented a means to extend the presence of family caregiving without replacing the human presence. This can be of reassurance against a common concern on the use of robotics solutions, relative to their risk of reducing the opportunities for human contact between older people and their families and/or caregivers [44]. From the point of view of the female primary user, the use of the robot has led to a greater sense of self-esteem, security and self-confidence, and also positively influenced the sense of independence and adequacy in dealing with situations of daily life. The direct control of the female user on the management of the robot at home and in general the active involvement in the research project and their strong motivation, may have also had a positive effect on their sense of self-efficacy.

Secondary user As for the secondary user, the initial expectations on the use of the telepresence robot were confirmed especially in terms of usefulness of the system as an aid to remotely control the physical and health status of his parents. The ability to make immediately eye contact and to be able to explore the physical environment were highly appreciated by the secondary user who had the perception of having a tool able to help him in managing situations that even though not critical, still worried him because of the state of fragility of his parents. Over time, for the secondary user, the telepresence system acquired a significant added value in order to maintain contacts that were judged true and effective. Overall, the telepresence system has become a valid support that is also somehow missed after the closure of the test site. The utility acknowledged and attributed to the system appears not to have suffered from poor usability perceived by the secondary user with respect to the interface and some functions/commands that are not intuitive in time. The docking operation had a strong initial impact on the adoption and consistent use of the telepresence system, at least during the first few months of use. The importance given to the system as a valuable aid to make immediately eye contact with the end user has suffered the effects of poor quality video of the webcam. In terms of psychosocial impact, the use of the system as a means of communication and remote control of parents, led to a significant improvement of the ability to share activities together with the primary users. Probably being involved in this experience with their parents, may have created a greater sense of sharing and greater empathy while raising also his sense of usefulness and efficiency in the management of the fragility of his family.

This result, even if it just refer to a case study, is in line with the idea that technology can be a support to strengthen the social inclusion of older people, facilitating the possibilities for communication and establishing eye contact reducing the impact of geographical distance, with friends and family. The potential of technology to support the network of social and emotional relationships is positively evaluated especially when it allows not only to hear each other but also to see each other and to have the feeling of being together [45-49].

Additionally, in this case the technology is not seen as a substitute of human care and contact, rather it represents a means to improve and enrich the ways of communication. 


\subsection{Readiness Level of the Telepresence Robot}

As previously mentioned, when performing long-term experiments, special attention should be dedicated to technological aspects connected to the prolonged use of robotics solution in real contexts. There is quite an amount of work to be done in creating robust contexts of use for telepresence robotic systems and some general comments can be derived from the experience related to the long-term usage described above.

Broadly speaking, from the primary users perspective, one of the main concerns in managing robotic solutions is related to the system being difficult to learn and maintain (e.g., [50]). Nevertheless, older adults have demonstrated a willingness to use such technology, when the goal is assistance for their independent living [11,51]. In the case of telepresence systems it is also important that secondary users, who teleoperate the robot, are facilitated in such operation. It is important to allow them to focus attention on the assistive interaction with the old person and, thus, experiencing an increasing (and easy) projection/immersion in the environment.

It is worth observing how a telepresence robot is a relatively simple system. It is completely operated by the remote user and thus it is usually not endowed with autonomous behaviors. However, in our work for fielding the GIRAFF in operational contexts we gathered incremental evidence that situations exist in which some technical advancements usually connected with autonomous behavior can enhance the robustness of the whole system in an application area where frail users are involved. For example, the robot should be able to act autonomously when the operator cannot control the telepresence robot properly, or when data transmission is lost. Additionally, from the secondary users' point of view, autonomous behaviors can increase their projection capability and achieve a safe and reliable operation of the telepresence robot in a (potentially) dynamic environment [52].

In this subsection, we present some contextualized use cases for autonomous capabilities that have been derived from the long-term deployment experience. Even though they have been elicited after experiments with the Giraff robot, most of them constitute a reference for enhancing a generic telepresence robotic platform.

Robot encumbrance One situation, quite common in Mediterranean countries, is related to the small size of apartments where old people live. The installation of telepresence robots such as the GIRAFF should take into account such limited spaces. Even skilled client users may have difficulties in controlling the robot within such small environments.

In this regard, the basic requirements needed to endow a robotic platform with autonomous navigation behaviors that could help overcoming these problems are the capabilities of: (a) mapping the environment, (b) localizing itself within a (partially) known environment and (c) avoiding the (possibly moving) obstacles. Usually, SLAM techniques [53] are exploited to deploy localization and mapping capabilities. Furthermore, many different solutions can be used to implement obstacle avoidance functionalities [54]. Additionally, the system should be equipped with some autonomous navigation ability to safely guide the telepresence robot to some specific home locations requested by the operator. For instance, the operator may request the telepresence robot to reach the kitchen in the apartment so that she/he can visually check the status of the stoves.

Docking A crucial location for the robot at home resulted to be the docking station used to recharge batteries and park it if idle. In fact, since the GIRAFF must not be left out of the docking station without control, this is the most important location and the robot should always be able to reach it. The robot should also be able to detect the status of its battery and, whenever its level is below a given threshold, automatically reach the docking station. Indeed, the return to docking is considered the GIRAFF's aspect that requires the synthesis of new solutions. Again here some autonomous capabilities would be of help.

Connectivity Another important issue of a long-term domestic test site is that very often it is not possible to rely on a continuous and stable WiFi internet connection (in particular, this issue has been detected as quite critical in Italy). Sometime, sudden communication breakdowns may leave the robot still with no active control. This is highly risky since the robot may remain stuck in an unsafe position also preventing the movements of the old person at home. In this case, whenever the data transmission is lost, the robot should automatically reach and, then, plug at the docking station, minimizing the risk to leave the GIRAFF with no charge in the middle of an apartment.

People searching During an emergency call ${ }^{4}$ a client user should take control of the robot and find the elderly as soon as possible in order to check his/her conditions. In such cases, the GIRAFF platform could be endowed with the capability of autonomously looking for the elder in the apartment instead of requiring the operator to (potentially) visit the whole house at random. Then, once the operator takes the control of the robot, it would automatically find a proper position to start a new dialogue, minimizing the movement of the robot in the environment and increasing the effectiveness of the interaction.

People following During a dialogue, the secondary user should be focused on the interaction with the old person

\footnotetext{
4 This is a specific capability that allows to force the usual operational functionalities. It enables an authorized client to bypass the old person authorization and connect with the home environment.
} 
avoiding to continuously control the robot for adjusting its position. Then, an interesting feature would be to enable GIRAFF to automatically identify the position of the old person and autonomously adapt to it (for example maintaining the old person centered on the robot camera).

Safety A final point of paramount importance is the safety of platform basic movements so as to avoid any scaring movement in proximity of the old person. In this respect recent technology improvements for fault-free low level control behaviors like those described in $[55,56]$ can potentially result very useful if integrated in telepresence systems.

It is worth observing how all these identified cases can be addressed with a smart integration of functionalities that are within the state-of-the-art of current autonomous robotics. Overall, the incremental integration of the features discussed above within "simple telepresence system" would allow to maintain a level of simplicity for the robotic platform (and in turn to still promote the level of acceptance) but also to overcome some of the encountered problems of long-term usage.

\section{Conclusions}

This article introduced a methodology, called MARTA, (Multidimensional Assessment of telepresence RoboT for older Adults) for the long-term study of telepresence robot's adaptivity and compatibility in the field of support to social interaction of elderly people. The pursued idea is to identify a set of relevant variables that are monitored over time according to a plan that can adapt to the different needs of the case study in order to understand the user's experience, attitude, interaction behaviors, acceptance and beliefs towards a technological solution and how they impact the adoption and effective daily use of a telepresence robot in a real context of daily life. The proposed approach also puts the emphasis on the need to follow a protocol for the deployment and maintenance of the technology itself that should be robust enough so as to minimize any problem related to technical malfunctions that may have a negative effect on the study of human-robot interaction. The method was evaluated on an ecological long-term case study with an installation and continuous use of the GIRAFF telepresence robot in the house of a couple of older adults. The results of the case study validate the proposed methodology and also are in line with the idea of using of robotic telepresence solution as a means to support empathic communication and social presence. It is worth highlighting how the prolonged experience supports the idea that technology is not to be intended as a substitute of human contacts and care, rather it can help ensuring a reacher and more intensive way of communication. The prolonged use of the technology in a real environment has also highlighted a number of possible technical improvements that can help make the real deployment of telepresence robots more fluid, robust and accepted, that can be directly imported by stateof-the-art results in robotics.

Our experience also confirms the importance of adopting and adapting a mixed methods approach for a long-term assessment aimed to achieve a deeper understanding of the environmental, technical and psychological factors that can influence the adoption, acceptance and effective use of a telepresence robot into the daily routine and living environment of people, once the effect of novelty and curiosity has disappeared.

It is also worth observing that the proposed approach can also be seen as a general framework that could potentially be used in and adapted for different contexts where the need exists to assess technology over long periods of time. In fact MARTA can be seen as a reference to be adapted and adjusted (e.g., adding new variables of interests or selecting the most appropriate ones in the different evaluation phases) so as to capture the specificity of new contexts and cases of interest.

In conclusion, we can say that this experience of long term case study analysis on the use of the GIRAFF robot in a domestic context encourages pursuing a user-centered approach that points a "magnifying glass" on the Technology - Environment - Person relationship established during a real-word prolonged experience of use.

Acknowledgments Authors have been collaborating with the Project ExCITE inside the EU Ambient Assisted Living Joint Program (AAL2009-2-125). Authors are indebted to partners of that project for the stimulating work environment. Interactions with the colleagues from Örebro University have been fruitful to refine the evaluation plan. The authors would like to dedicate this work to Silvia Coradeschi for her commitment in the ExCITE project.

Open Access This article is distributed under the terms of the Creative Commons Attribution 4.0 International License (http://creativecomm ons.org/licenses/by/4.0/), which permits unrestricted use, distribution, and reproduction in any medium, provided you give appropriate credit to the original author(s) and the source, provide a link to the Creative Commons license, and indicate if changes were made.

\section{References}

1. Tapus A, Matarić MJ, Scassellati BB (2007) The grand challenges in socially assistive robotics. ieee robot automa mag 14(1):35-42

2. Pineau J, Montemerlo M, Pollack M, Roy N, Thrun S (2003) Towards robotic assistants in nursing homes: challenges and results. Robot Auton Syst 42(3-4):271-281

3. Cesta A, Cortellessa G, Rasconi R, Pecora F, Scopelliti M, Tiberio L (2011) Monitoring elderly people with the RoboCare Domestic Environment: interaction synthesis and user evaluation. Comput Intel 27(1):60-82

4. Saffiotti A (2009) The concept of Peis-ecology: integrating robots in smart environments. Acta Futura 3:35-42

5. Coradeschi S, Cesta A, Cortellessa G, Coraci L, Gonzalez J, Karlsson L, Furfari F, Loutfi A, Orlandini A, Palumbo F, Pecora F, von 
Rump S, Stimec A, Ullberg J, Ostlund B (2013) GiraffPlus: combining social interaction and long term monitoring for promoting independent living. In: HSI: The 6th international conference on human system interaction, IEEE, pp 578-585

6. Cavallo F, Limosani R, Manzi A, Bonaccorsi M, Esposito R, Di Rocco M, Pecora F, Teti G, Saffiotti A, Dario P (2014) Development of a socially believable multi-robot solution from town to home. Cognit Comput 6(4):954-967

7. Di Nuovo A, Broz F, Belpaeme T, Cangelosi A, Cavallo F, Esposito R, Dario P (2014) A web based Multi-Modal Interface for elderly users of the Robot-Era multi-robot services. In: IEEE (Ed.), international conference on systems, man and cybernetics (SMC), pp 2186-2191

8. Lee MK, Takayama L (2011) Now, I have a body: uses and social norms for mobile remote presence in the workplace. In: Proceedings of the 2011 annual conference on human factors in computing systems, CHI'11. ACM, New York, pp 33-42

9. Tsui KM, Desai M, Yanco HA, Uhlik C (2011) Exploring use cases for telepresence robots. In: Proceedings of the 6th intrenational confernce on human-robot interaction, HRI '11. ACM, New York, pp 11-18. doi:10.1145/1957656.1957664

10. Beer JB, Takayama L (2011) Mobile remote presence for older adults: acceptance, benefits, and concerns. In: Proceedings of human robot interaction: HRI 2011. Lausanne, $\mathrm{CH}$, pp 19-26

11. Cesta A, Cortellessa G, Giuliani M, Pecora F, Scopelliti M, Tiberio L (2007) Psychological implications of domestic assistive technology for the elderly. PsychNol J 5(3):229-252

12. Cesta A, Coradeschi S, Cortellessa G, Gonzalez J, Tiberio L, Von Rump S (2010) Enabling social interaction through embodiment in ExCITE. In: ForItAAL. Second Italian forum on ambient assisted living

13. Coradeschi S, Loutfi A, Kristoffersson A, Von Rump S, Cesta A, Cortellessa G (2011) Towards a methodology for longitudinal evaluation of social robotic telepresence for elderly. In: Human-Robot interaction workshop on social robotic telepresence

14. Hüttenrauch H, Eklundh KS (2002) Fetch-and-carry with CERO: observations from a long-term user study with a service robot. In: IEEE (Ed.), Proceedings of robot and human interactive communication. 11th IEEE international workshop on IEEE, pp 158-163

15. Marti P, Pollini A, Rullo A, Shibata T (2005) Engaging with artificial pets. In: Proceedings of the 2005 annual conference on european association of cognitive ergonomics. University of Athens, Athens, pp 99-106

16. Kanda T, Sato R, Saiwaki N, Ishiguro H (2007) A two-month field trial in an elementary school for long-term Human-Robot interaction. IEEE Trans Robot 23(5):962-971

17. Kim H, Lee H, Chung S, Kim C (2007) User-centered approach to path planning of cleaning robots: analyzing user's cleaning behavior. In: ACM, New York (Ed.) Proceedings of the ACM/IEEE international conference on Human-Robot interaction, pp 373-380

18. Lee H, Kim HJ, Kim C (2007) Autonomous behavior design for robotic appliance. In: IEEE (Ed.), 2nd ACM/IEEE international conference on Human-Robot interaction-HRI2007, pp 201-208

19. Mutlu B, Forlizzi J (2008) Robots in organizations: the role of workflow, social, and environmental factors. In: IEEE (Ed.) 3rd ACM/IEEE international conference on Human-Robot interaction. (HRI), pp 287-294

20. Young JE, Sung J, Voida A, Sharlin E, Igarashi T, Christensen HI, Grinter RE (2011) Evaluating Human-Robot interaction. Int J Soc Robot 3(1):53-67

21. Sung JY, Grinter RE, Christensen HI (2010) Domestic robot ecology an initial framework to unpack long-term acceptance of robots at home. Int J Soc Robot 2(4):417-429

22. Wada K, Shibata T (2007) Living with seal robots-its sociopsychological and physiological influences on the elderly at a care house. IEEE Trans Robot 23(5):972-980
23. Marti P, Bacigalupo M, Giusti L, Mennecozzi C, Shibata T (2006) Socially assistive robotics in the treatment of behavioural and psychological symptoms of dementia. In: IEEE (Ed.), The First IEEE-RAS-EMBS international conference on biomedical robotics and biomechatronics. BioRob 2006, pp 483-488

24. Sabelli A, Kanda T, Hagita N (2011) A conversational robot in an elderly care center: an ethnographic study. In: ACM, New York (ed) Proceedings of the 6th international Conference on Human-Robot interaction, pp 37-44

25. Salter T, Dautenhahn K, Bockhorst R (2004) Robots moving out of the laboratory-detecting interaction levels and human contact in noisy school environments. In: ROMAN (ed.) Proceedings of the 13th IEEE international workshop on robot and human interactive communication-ROMAN, pp 563-568

26. Gockley R, Bruce A, Forlizzi J, Michalowski M, Mundell A, Rosenthal S (2005) Designing robots for long-term social interaction. In: IEEE (ed) Proceedings of IEEE/RSJ international conference on intelligent robots and systems, (IROS 2005), pp $1338-1343$

27. Leite I, Martinho C, Paiva A (2013) Social robots for long-term interaction: a survey. Int J Soc Robot 5(2):291-308

28. Sung J, Christensen H, Grinter R (2009) Robots in the wild: understanding long-term use. In: ACM, New York (ed.) Proceedings of the 4th ACM/IEEE international conference on Human-Robot interaction, pp 45-52

29. Fernaeus YH, Jacobsson M, Ljungblad S (2010) How do you play with a robotic toy animal? A long-term study of pleo. In: ACM, New York (ed) Proceedings of the 9th international conference on interaction design and children, pp 39-48

30. Klamer T, Ben Allouch S, Heylen D (2011) Adventures of harveyuse, acceptance of and relationship building with a social robot in a domestic environment. In: Human-Robot personal relationships LNCS, vol 59. Springer, Berlin, pp 74-82

31. Ganster T, Eimler SC, von der Pütten AM, Hoffmann L, Krämer NC (2010) Methodological considerations for long-term experience with robots and agents. In: Proceedings of EMCSR 2010

32. Tanaka F, Cicourel A, Movellan J (2007) Socialization between toddlers and robots at an early childhood education center. Proc Nat Acad Sci 104:17954-17958

33. Leite I, Martinho C, Pereira A, Paiva A (2009) As time goes by: long-term evaluation of social presence in robotic companions. In: 18th IEEE international symposium on robot and human interactive communication. RO-MANIEEE, New York, pp 669-674

34. Russell DW (1996) Ucla loneliness scale (version 3): reliability, validity, and factor structure. J Personal Assess 66:20-40

35. Ware JEJ, Kosinski M, Keller SD (1996) A 12-item short-form health survey: construction of scales and preliminary tests of reliability and validity. Medical Care 34(3):220-233

36. Zimet GD, Dahlem NW, Zimet SG, Farley GK (1988) The multidimensional scale of perceived social support. J Personal Assess 52(1):30-41

37. Yesavage JA, Brink TL, Rose TL, Lum O, Huang V, Adey M, Leirer VO (1983) Development and validation of a geriatric depression screening scale: a preliminary report. J Psychiatr Res 17(1):37-49

38. Terracciano A, McCrae RR, Costa PT (2003) Factorial and construct validity of the Italian Positive and Negative Affect Schedule (PANAS). Eur J Psychol Assess Official Organ Eur Assoc Psychol Assess 19(2):131-141

39. Sauro J, Lewis J (2012) Quantifying the user experience: practical statistics for user research. In: Software Usability Scale (SUS), Ch 8. Morgan Kaufmann, pp 198-208

40. Heerink M, Kröse BJA, Evers V, Wielinga BJ (2010) Assessing acceptance of assistive social agent technology by older adults: the Almere model. I J Soc Robot 2(4):361-375

41. Jutai HDJ (2002) Psychosocial impact of assistive devices scale (piads). Technol Disabil 14(3):107-111 
42. Lombard M, Ditton T, Weinstein L (2009) Measuring telepresence: the temple presence inventory. In: Proceedings of the 12th international workshop on presence. Los Angeles, San Francisco

43. Patton M (1990) Qualitative evaluation and research methods. Sage, Beverly Hills

44. Sharkey A, Sharkey N (2010) Granny and the robots: ethical issues in robot care for the elderly. Ethics Inf Technol 14(1):27-40

45. Bradley N, Poppen W (2003) Assistive technology, computers and internet may decrease sense of isolation for homebound elderly and disabled persons. Technol Disabil 15:19-25

46. Czaja SJ, Nair SN (2006) Human factors engineering and systems design. In: Salvendy G (ed) Handbook of human factors and ergonomics, vol 3, 3rd edn. Wiley, Hoboken, pp 32-53

47. Magnusson L, Hanson E, Borg M (2004) A literature review study of information and communication technology as a support for frail older people living at home and thier family carers. Technol Disability 16:223-235

48. Moser CV, Neureiter K, Sellner W, Tscheligi M (2011) Elderly's social presence supported by ICTs. Investigating user requirements for social presence. In: IEEE international conference on privacy, security, risk, and trust, pp 738-741

49. Savolainen L, Hanson E, Magnusson L, Gustavsson T (2008) An internet-based videoconferencing system for supporting frail elderly people and their carers. J Telemed Telecare 14(2):79-82

50. Demiris G, Rantz M, Aud M, Marek K, Tyrer H, Skubic M, Hussam A (2004) Older adults attitudes towards and perceptions of smart home technologies: a pilot study. Med Inf Internet Med 29(2):8794. doi:10.1080/14639230410001684387

51. Sharit J, Czaja SJ, Perdomo D, Lee CC (2004) A cost-benefit analysis methodology for assessing product adoption by older user populations. Appl Ergon 35(2):81-92. doi:10.1016/j.apergo.2003. 12.003

52. Tsai TC, Hsu YL, Ma AI, King T, Wu CH (2007) Developing a telepresence robot for interpersonal communication with the elderly in a home environment. Telemed e-Health 13(4):407-424

53. Smith R, Self M, Cheeseman P (1990) Estimating uncertain spatial relationships in robotics. Springer-Verlag New York, Inc., New York, pp 167-193 http://dl.acm.org/citation.cfm?id=93002.93291

54. Khatib O (1986) Real-time obstacle avoidance for manipulators and mobile robots. Int J Rob Res 5, 90-98. doi:10.1177/ 027836498600500106 . http://dl.acm.org/citation.cfm?id=6806. 6812
55. Bensalem S, de Silva L, Gallien M, Ingrand F, Yan R (2010) "Rock Solid" Software: a verifiable and correct-by-construction controller for rover and spacecraft functional levels. In: i-SAIRAS10. Proceedings of the 10th international symposium on artificial intelligence, robotics and automation in space

56. de Silva L, Yan R, Ingrand F, Alami R, Bensalem S (2015) A verifiable and correct-by-construction controller for robots in human environments. In: Proceedings of the 10th annual ACM/IEEE international conference on Human-Robot interaction extended abstracts, HRI'15 extended abstracts, pp. 281-281. ACM, New York. doi:10.1145/2701973.2702098. http://doi.acm.org/10.1145/ 2701973.2702098

Amedeo Cesta [M.S. Electronic Engineering 1983, Ph.D. Computer Science 1992] is a Senior Research Scientist in Artificial Intelligence and Group Leader at CNR-ISTC. He has conducted research in several areas of Artificial Intelligence like Multi-Agent Systems, Intelligent Interaction and Automated Planning \& Scheduling, and pursued the synthesis of innovative Cognitive Systems in several projects.

Gabriella Cortellessa [M.S. Engineering Computer Science 2001, Ph.D. Cognitive Psychology 2005] is a research scientist at CNR-ISTC. She is an AI researcher with experience in user interactive and personalized systems, methods for evaluating intelligent systems, human-robot interaction.

Andrea Orlandini [M.S. Computer Science Engineering 2002, Ph.D. Computer Science and Automation 2006] is a technologist at CNRISTC. His research interests spans over automated planning, dependable plan execution and model-based robot control.

Lorenza Tiberio [M.S. Clinical Psychology 2005, Ph.D. Cognitive Psychology, Psychophysiology and Personality 2014] has been a research assistant at CNR-ISTC from 2008 to 2014. She is now an independent consultant in human factors and human-robot interaction. Her research interests include clinical neuropsychology, psychology of aging, processes of technology acceptance, and assistive robotics assessment. 